\title{
Research Paper \\ A Comparative Study On Emotion Regulation Of Older And Younger Adults
}

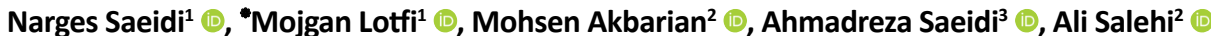

1. Department of Mental Health, School of Behavioral Sciences and Mental Health (Tehran Institute of Psychiatry), Iran University of Medical Sciences, Tehran, Iran. 2. Department of Health Psychology, School of Behavioral Sciences and Mental Health (Tehran Institute of Psychiatry), Iran University of Medical Sciences, Tehran, Iran. 3. Department of Psychology and Educational Sciences, School of Psychology and Educational Sciences, University of Allameh Tabataba'i, Tehran, Iran.

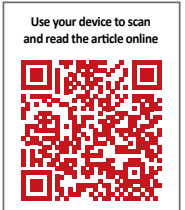

Cittation: Saeidi N, Lotfi M, Akbarian M, Saeidi A, Salehi A. [A Comparative Study On Emotion Regulation Of Older And Younger Adults (Persian)]. Iranian Journal of Ageing. 2021; 16(3):412-425. http://dx.doi.org/10.32598/sija.2021.16.3.3153.1

doi) $h$ http://dx.doi.org/10.32598/sija.2021.16.3.3153.1

\section{(i) (8)}

Received: 01 Feb 2021 Accepted: 24 Apr 2021 Available Online: 01 Oct 2021

Keywords:

Emotional regulation, Mental health, Older adults

\section{ABSTRACT}

Objectives Healthy aging is a natural human right and emotion regulation is an important factor in improving mental health promotion in different developmental ages. This study aims to compare emotion regulation in older and younger adults.

Methods \& Materials This is a causal-comparative study. The study population includes all older and younger adults in Tehran in 2019. Of these, 235 were selected using a convenience sampling method. They completed Difficulties in Emotion Regulation Scale (DERS), Emotion Regulation Questionnaire (ERQ), Mentalized Affectivity Scale (MAS), and Emotion Regulation of Others and Self (ERO and ERS) questionnaires. Data analysis was performed using multivariate analysis of variance, independent t-test, and paired t-test in SPSS v. 20 software.

Results The scores of DERS ( $F=17.08, P<0.001)$, ERQ ( $F=16.07, P<0.001)$, and ERS $(t=-12.2, P=0.35)$ were significantly higher in older adults than younger adults, while there was no significant difference between them in MAS $(t=-1.29, P=0.198)$ and $E R O(F=0.256, P=0.614)$ scores.

Conclusion The scores of DERS, ERS, and cognitive reappraisal subscale of ERQ are higher in older adults, but there is no significant difference between older and younger adults in the scores of MAS and ERO. Mental health professionals can take effective measures at the community level based on these findings to promote emotional health and psychological well-being of older people.

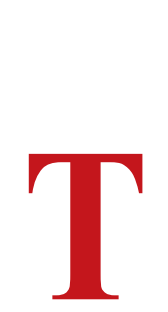

Extended Abstract

\section{Introduction}

oday, the global population is aging [1]. In Iran, along with the phenomenon of population aging, it is necessary to create conditions for better adaptation of the elderly to the living environment in order to make various plans in the physical, psychological and social ar- eas appropriate to this group [2]. Healthy aging is the right of all human beings [3]. Emotion regulation is one of the effective factors in promoting mental health [4]. Considering that the development of emotion regulation strategies in the life span continues uninterruptedly [5], and adaptive emotion regulation is associated with mental health and maladaptive emotion regulation is associated with psychological pathology [6], studies have shown that the elderly have higher emotional well-being than young adults due to the use of more adaptive strategies [7, 8]. In this study, an

* Corresponding Author:

Mojgan Lotfi, PhD.

Address: Department of Mental Health, School of Behavioral Sciences and Mental Health (Tehran Institute of Psychiatry), Iran University of Medical Sciences, Tehran, Iran.

Tel: +98 (21) 66551655

E-mail: lotfi.mo@iums.ac.ir 
attempt is made to compare the emotional regulation patterns in the elderly and adults in order to take more effective educational measures in accordance with the age of individuals to promote emotional health and mental well-being in the community.

\section{Methods}

This is a casual/comparative study with an ethical approval from the Ethics Committee of Iran University of Medical Sciences (Code: IR.IUMS.REC.1398.955). Participants were 235 older and young adults. The older adults were selected from neighborhood houses and young adults from health centers in Tehran, Iran in 2019. The sample size was calculated 233 using the formula [9] where the standard deviation was 3.9, the mean effect size (d) was 0.5 and $\alpha=0.05$ [10]. However, 235 were recruited using a convenience sampling method. Due to lack of access to the elderly during the COVID-19 pandemic, most of participants were young adults. Inclusion criteria were age $\geq 60$ years for the elderly and $20-59$ years for adults, verbal consent, literacy, ability to understand the questions, cognitive health, and no history of specific mental and physical disorders. Exclusion criteria were lack of cooperation and inability to understand the questions. For collecting data from the elderly, we visited the neighborhood houses and for adults, we visited health centers. After providing explanations about the research conditions to the participants and the confidentiality of their information, the research process began by completing a demographic form (surveying age, gender and education) and Difficulties in Emotion Regu- lation Scale (DERS), Emotion Regulation Questionnaire (ERQ), Mentalized Affectivity Scale (MAS), and Emotion Regulation of Others and Self (ERO and ERS). The reliability of the subscales of these questionnaires were high (0.63 to 0.92), except for the total score of the ERS (0.63) and extrinsic affect-worsening subscale score of the ERO (0.65) which were low. Data were analyzed in SPSS v. 22 software using the descriptive statistics, MANOVA to compare the scores of DERS, ERQ and ERO, independent t-test to compare the scores of ERS and MAS, and paired t-test was used to compare cognitive reappraisal and suppression in each group. Normality test was performed using Shapiro-Wilk test.

\section{Results}

Young adults included $42.1 \%$ men and $57.9 \%$ women, and older adults included $46.1 \%$ men and $53.9 \%$ women. Educational level between older and young adults was significantly different based on chi-square test $(p<0.05)$, but there was no significant difference between them in terms of gender. In the study of normality of data distribution for groups in all variables, results showed a normal distribution $(\mathrm{P}>0.05)$. The mean total scores of DERS, ERQ, ERO, ERS, and MAS were higher in older adults than in young adults. The correlation between MAS and DERS showed a significant negative relationship $(\mathrm{P}<0.05)$, which was higher in young adults than in the elderly. The relationship between MAS and other variables was significant and positive, where it had higher correlation with ERO and ERQ in the elderly, but its correlation with ERS was higher in

Table 1. Comparing the mean scores of study variables

\begin{tabular}{|c|c|c|c|c|c|c|}
\hline Variables & Group & Mean \pm SD & $\begin{array}{c}\text { Lower Bound } \\
\text { of } 95 \% \mathrm{Cl}\end{array}$ & $\begin{array}{c}\text { Upper Bound } \\
\text { of } 95 \% \mathrm{Cl}\end{array}$ & Sig. & Test \\
\hline DERS & $\begin{array}{l}\text { Young adults } \\
\text { Older adults }\end{array}$ & $\begin{array}{l}84.63 \pm 20.03 \\
95.80 \pm 17.89\end{array}$ & -16.49 & -5.84 & $>0.001$ & \\
\hline ERQ & $\begin{array}{l}\text { Young adults } \\
\text { Older adults }\end{array}$ & $\begin{array}{l}4.19 \pm 1.02 \\
4.76 \pm 0.97\end{array}$ & -0.84 & -0.28 & $>0.001$ & MANOVA \\
\hline ERO & $\begin{array}{l}\text { Young adults } \\
\text { Older adults }\end{array}$ & $\begin{array}{l}3.03 \pm 0.60 \\
3.07 \pm 0.49\end{array}$ & $-0.19-$ & 0.11 & 0.614 & \\
\hline MAS & $\begin{array}{l}\text { Young adults } \\
\text { Older adults } \\
\text { Young adults } \\
\text { Older adults }\end{array}$ & $\begin{array}{l}2.64 \pm 0.57 \\
2.77 \pm 0.30 \\
4.84 \pm 0.60 \\
4.93 \pm 0.43\end{array}$ & $-0.23-$ & -0.08 & 0.198 & $\begin{array}{c}\text { Independent } \\
\text { t-test }\end{array}$ \\
\hline
\end{tabular}


young adults. As presented in Table 1, the scores of DERS, ERQ and ERS showed a significant difference between the two groups. Regarding the variables of ERO and MAS, although their means were higher in the elderly, but this difference was significantly difference $(\mathrm{P}>0.05)$. Furthermore, the difference in the strategies of reappraisal and suppression between the two groups was significant $(\mathrm{P}<0.05)$ where the difference in cognitive reappraisal of both age groups was higher than in suppression.

\section{Discussion and Conclusion}

The scores of DERS, ERS, and cognitive reappraisal subscale of ERQ are higher in the elderly, but there is no significant difference between the elderly and young adults in ERO and MAS scores. The elderly are more likely to use adaptive emotion regulation and self-emotion regulation strategies. Therefore, some of the factors that affect emotion regulation in the life span are changing, while some are relatively fixed. In other words, given the use of adaptive emotion regulation strategies in old age, it can be said that some patterns of emotion regulation are modified at older ages and can lead to effective interpersonal relations, decision-making and adaptive behaviors. As a result, greater adjustment is due to better emotional regulation, which can be taken into account in the areas of interpersonal and intrapersonal emotion regulation, empathy and mutual understanding, relevant trainings to promote psychological well-being of all people, especially younger people.

\section{Ethical Considerations}

Compliance with ethical guidelines

This study is approved by the Ethics Committee of Iran University of Medical Sciences (Code: IR.IUMS. REC.1398.955).

\section{Funding}

This research did not receive any grant from funding agencies in the public, commercial, or non-profit sectors.

Authors' contributions

All authors equally contributed to preparing this article.

Conflicts of interest

The authors declared no conflict of interest. 


\author{
مقاله يُووهشى \\ تنظيم هيجانى: مطالعه مقايسهاي در سالمندان و بالغين

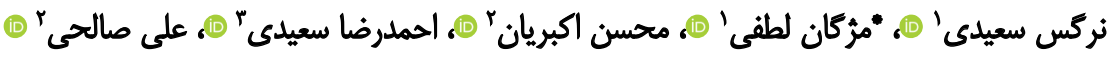 \\ I. كروه سلامت روان، داتشكده علوم رفتارى وسلامت روان (انستيتو روانيزشكى تهران)، دانشكاه علوميزشكى ايران، تهران، ايران.

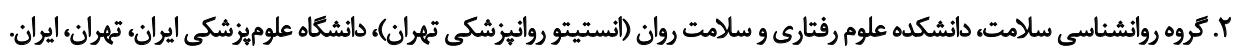

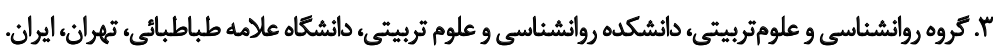

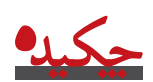

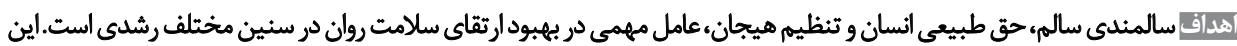

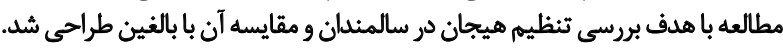

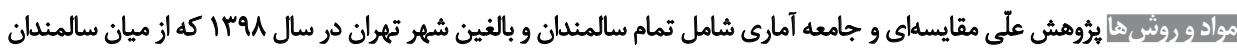

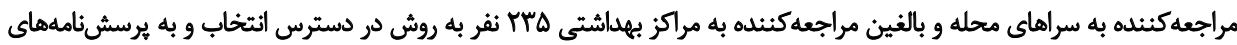

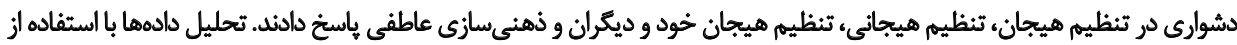

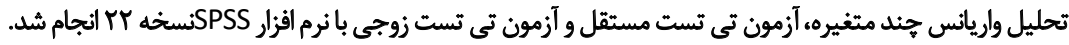

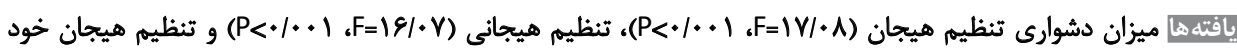

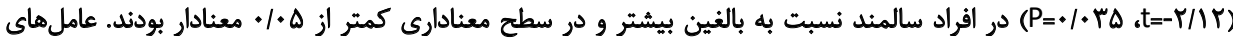

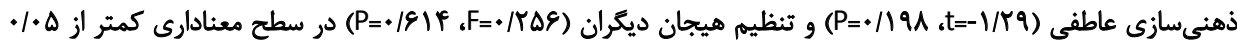

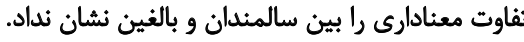

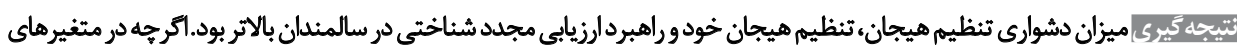

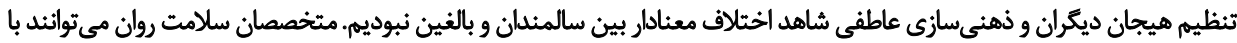

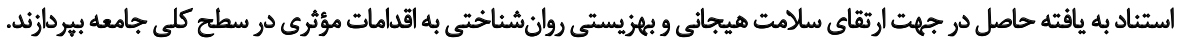

تاريخ دريافت: "rا بهمن

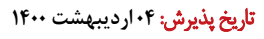

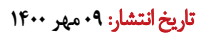

كليدوأرها:

تنظيم هيجان، سلامت روانى، سالمندان

همجنين بيشرفت علم يزشكى و بهبود شرايط بهداشتى شاهد

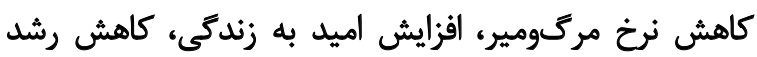

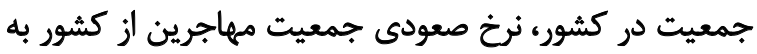

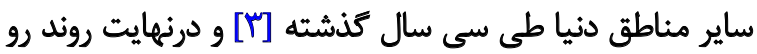

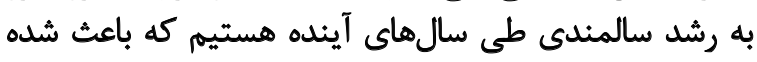

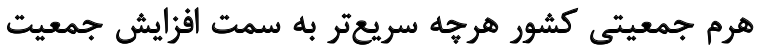
سالمندى حركت كند.

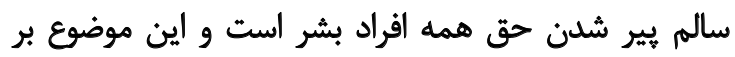

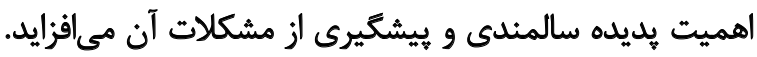

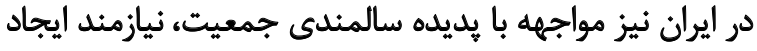

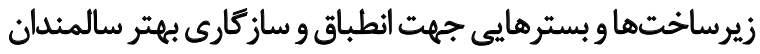
با محيط زندگى است كه لازم است در حيطهائى جسان جسمانى،

در حال حاضر جمعيت جهانى در عرصه سالمندى كام

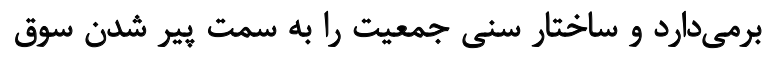

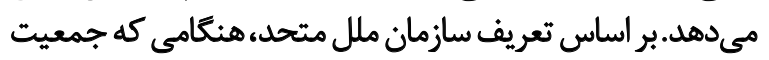

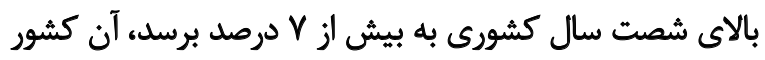

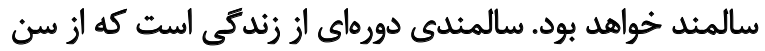

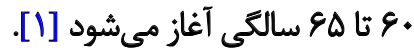

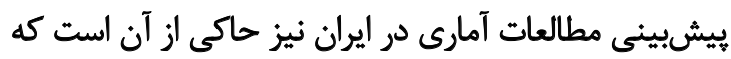

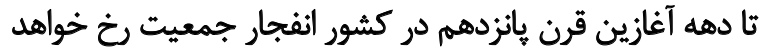

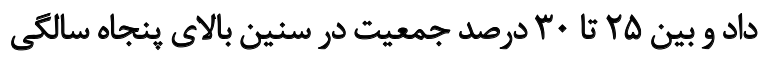

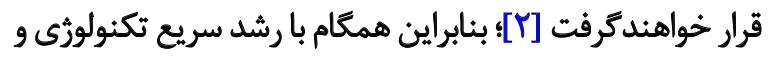


در اين راستا تئورى انتخاب اجتماعي بنيز بيشترين استدلال

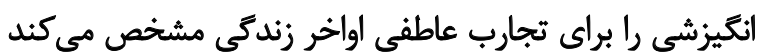

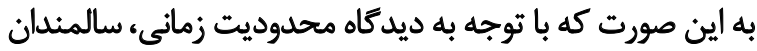

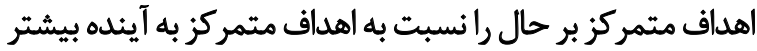

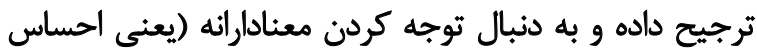

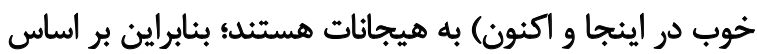

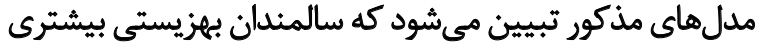
رانسبت به بالغين تجربه مي كنيند [11]. از سوى ديكر ، اكر جه نظريههاى متفاوتى براى كمك به بر درك ما

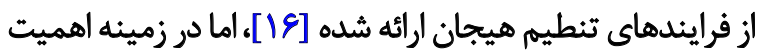
مفاهيم ذهنى إنى ونى و شكل

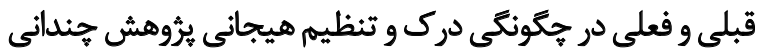

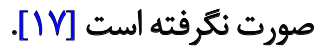

در هروهش حاضر به ارزيابى تنظيم هيجان بر اساس نظريه

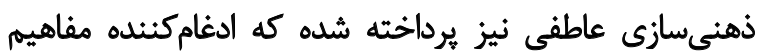

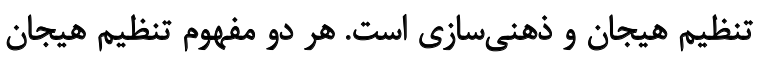

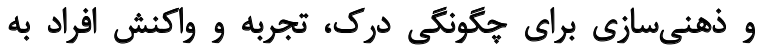

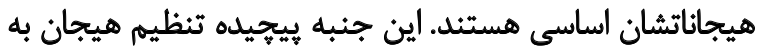

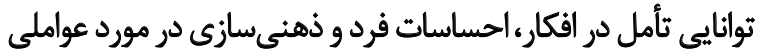

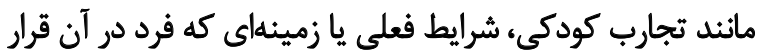

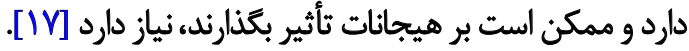
با نظر به موارد اشاره شده و با توجه به اينكه سالمندى سالمه،

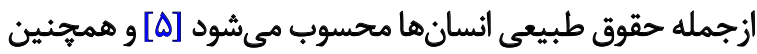

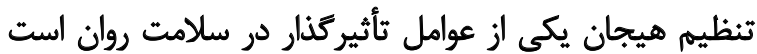

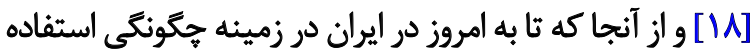

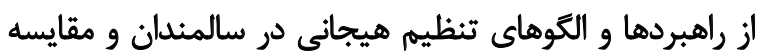

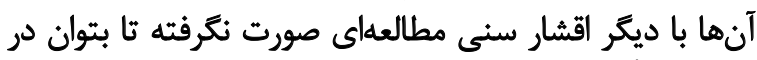

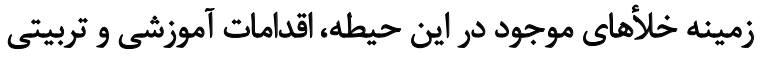

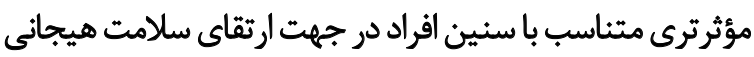

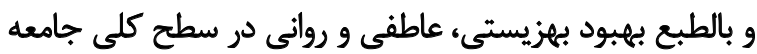

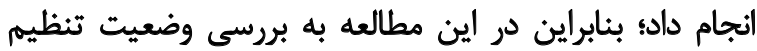
هيجان در سالمندان و مقايسه آن با بالغين يرداختهائ برايم.

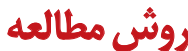

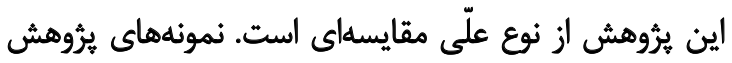

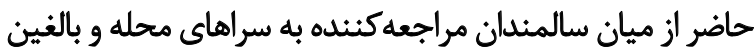

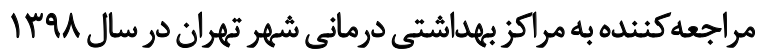

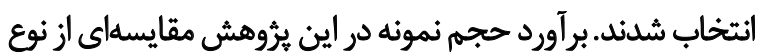

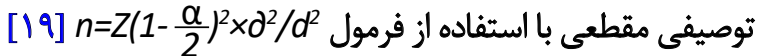

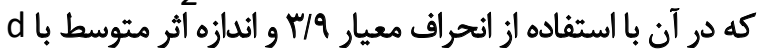

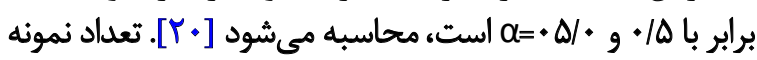

2. Socioemotional Selectivity Theory
روانشناختى، اجتماعى، فرهنگى و محيطى برنامهريزىهاي

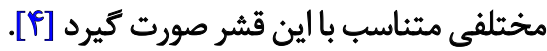
مطالعات نشان دادهاند كه سالمندان در مقايسه باديكر اقشار سنى

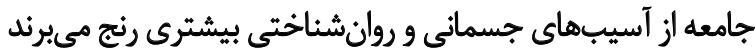

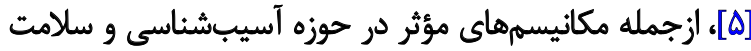

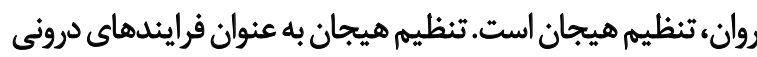

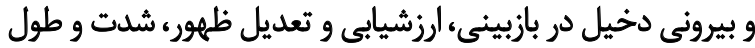

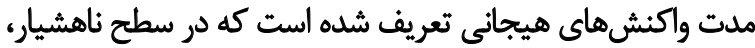
نيمه هشيار و هشيار به كار كرفته مي تعشود [ع]

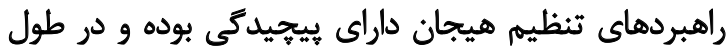

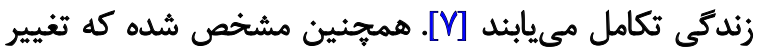

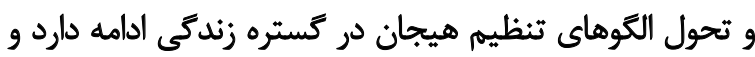

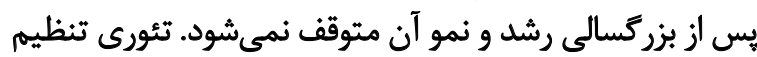

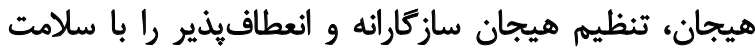

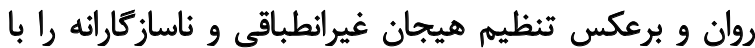

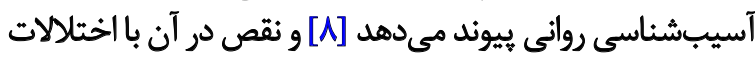

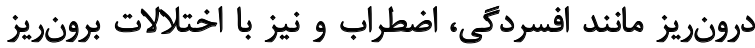

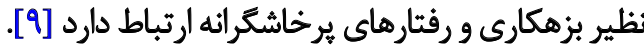
كاشدان و روتنبرى نيز نشان دادهاند افراد داراى نشانكان

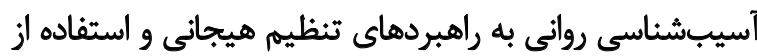
آنها دسترسى كمتر و نسبت به استفاده از آنها آنها انعطافيذئيرى

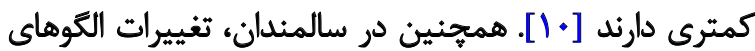

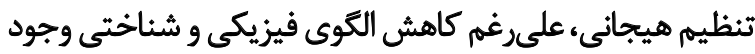

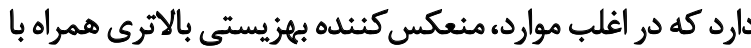

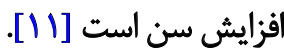

با كمال تعجب، تحقيقات طولى و مقطعى نشان دادهاند كه

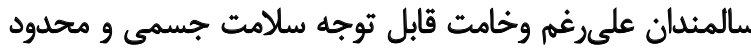

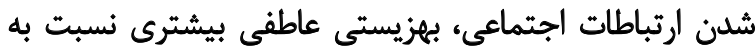

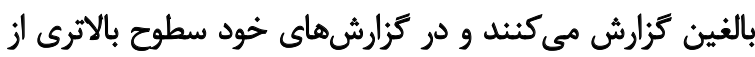

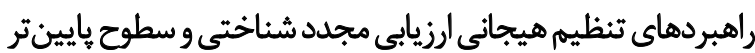

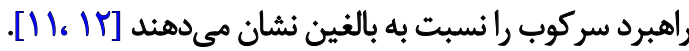

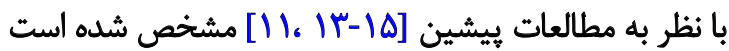

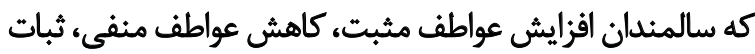

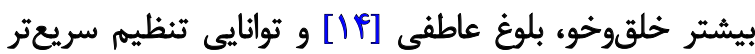

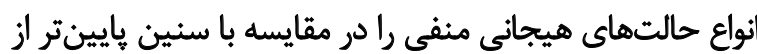

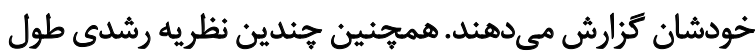

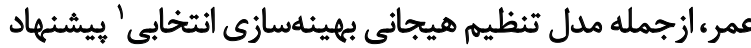

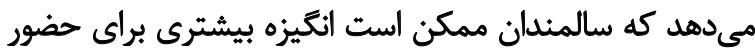

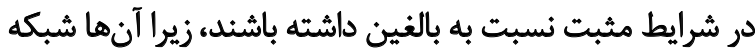

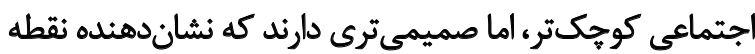

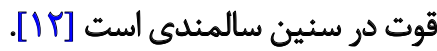

1. Selection, Optimization and Compensation 
در كنترل تكانه، فقدان آكاهى هيجانى، دسترسى محدود به

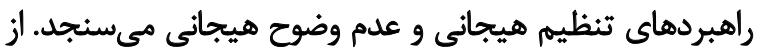

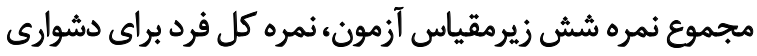
تنظيم هيجان محاسبه مي نيودي.

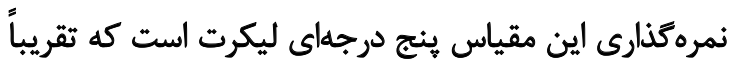

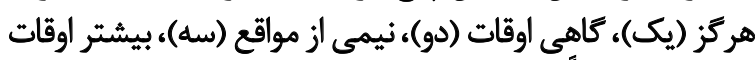

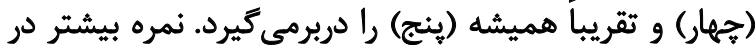

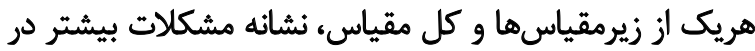

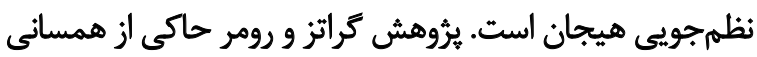

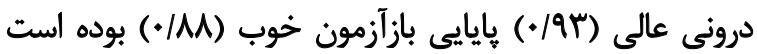

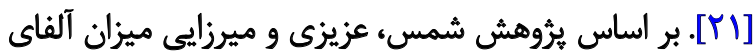

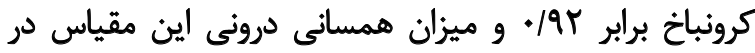

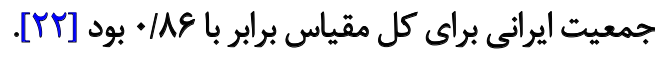

\section{يرسش ثامه تنظيم هيجاني ^}

كراس و جان يرسشنامه تنظيم هيجانى را تدوين كردهاند.

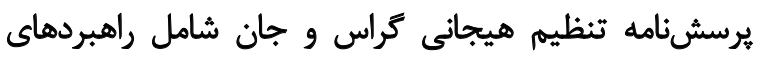

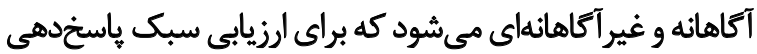

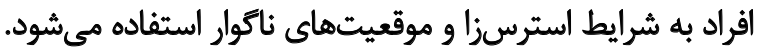

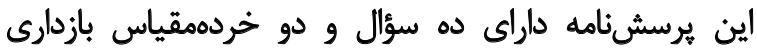

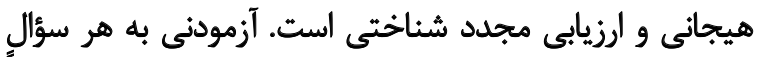

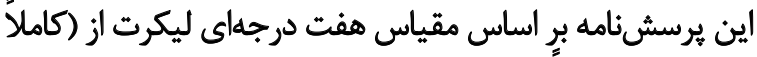

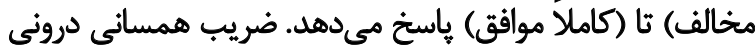

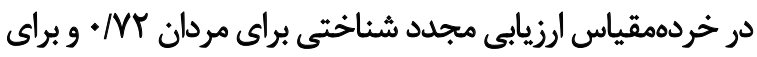

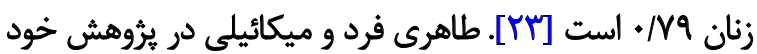

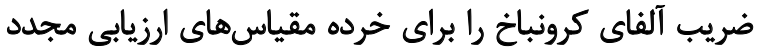

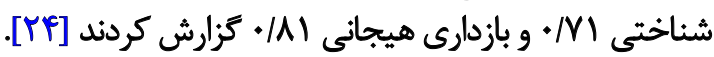

\section{يوسش نامه تنظظيم هيجان ديكران و خُود"}

هرسشانامه تنظيم هيجان خود و ديكـران، توسط نيون

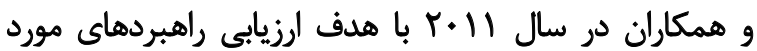

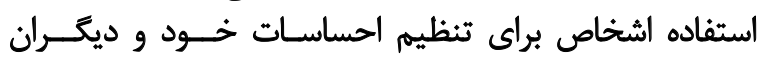

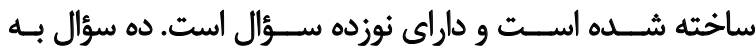

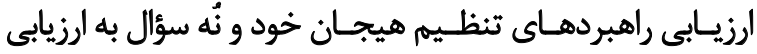

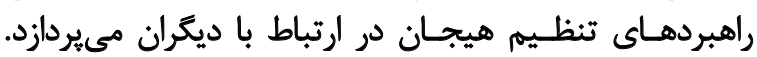

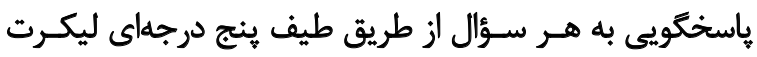
صـورتمى كيـرد.

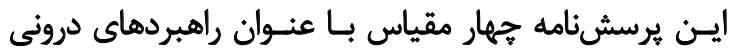

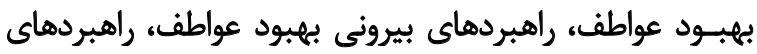

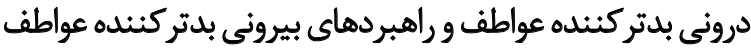

8. Emotion Regulation Questionnaire (ERQ)

9. Emotion Regulation of Others and Self (EROS)
حداقل كستr مورد برآورد شد و در يثوهش حاضر بر اساس روش

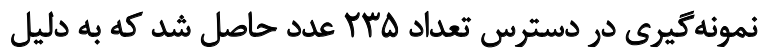
وجود مشكلاتى از قبيل عدم دسترسى برون به سالمندان در شرايط

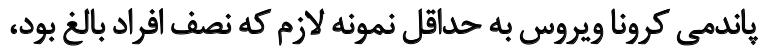
بسنده كرديم. معيارهاى ورود شامل شرط سنى شصت سال به بالا براى گروه

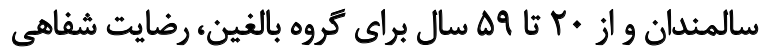

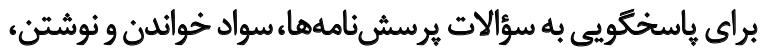

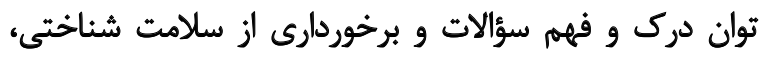

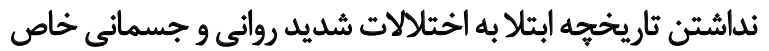

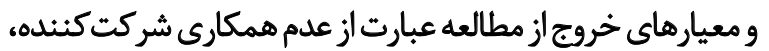

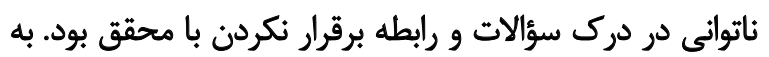

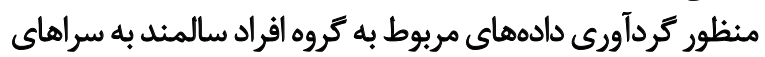

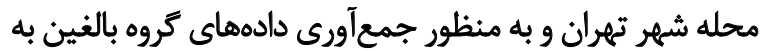
مراكز بهداشتى درمانى شهر تهران مراجعه شد.

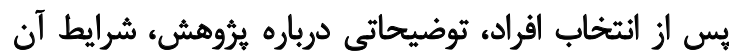

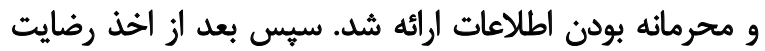

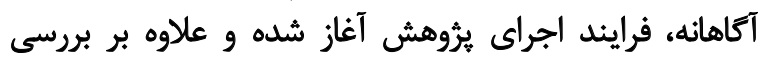

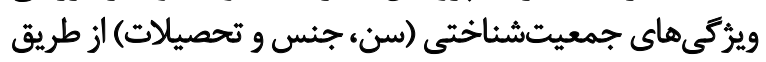

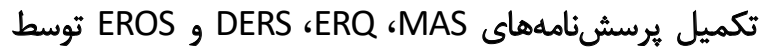

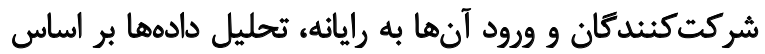

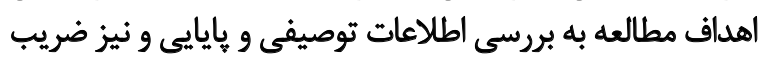
همبستكى بيرسون براى ارتباط بين عوامل مىيردازد. بله منظور مقايسه خردهمقياسها و عوامل در كروه سالمندان

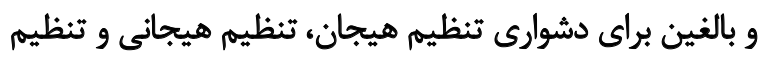

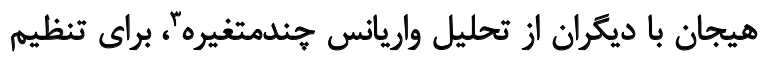

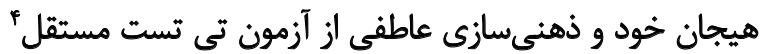

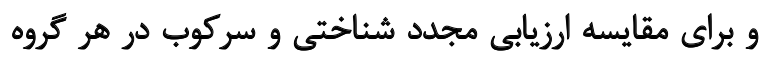

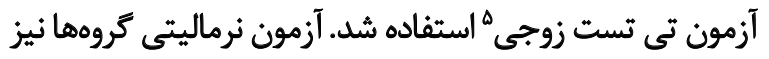

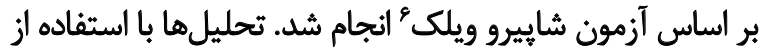

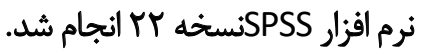

\section{مقياس دشوارى تنظيم هيبجان v}

كراتز و رومر براى سنجش دشوارى در تنظيم هيجانى مقياس

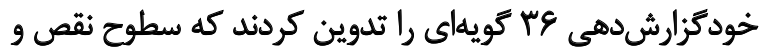

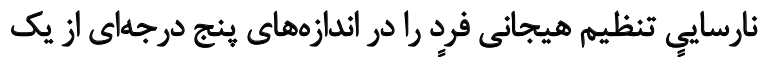

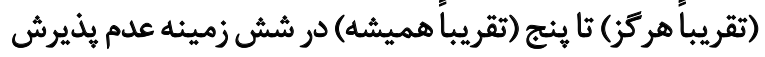

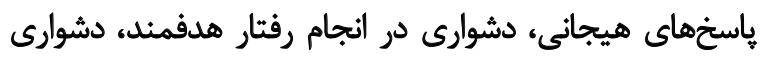

\section{MANOVA}

4. Independent T-Test

5. Paired T-Test

6. Shapiro-Wilk

7. Difficulties in Emotion Regulation Scale (DERS) 


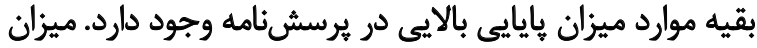

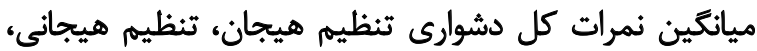

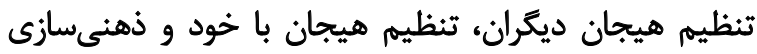

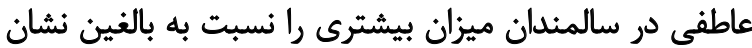

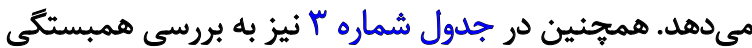
ييرسون بين عاملها در مطالعه يرداخته شدهاست. بر اساس جدول شماره ب در ارتباط بين متغيرهاي مورد مطالعه،

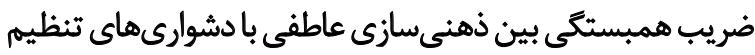

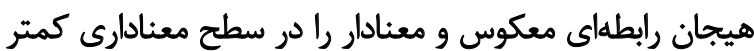

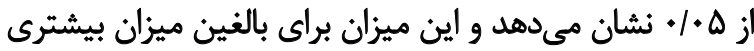

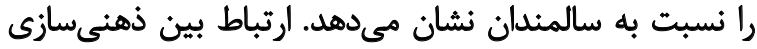

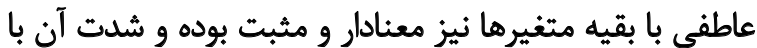

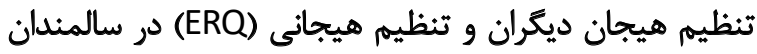

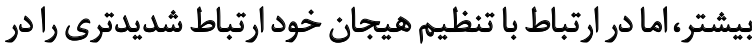

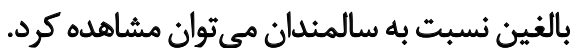
در جدول شماره F به مقايسه بين دشوارىهاى تنظيم هيجان،

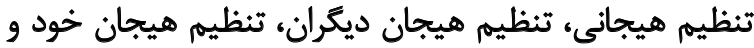

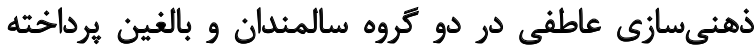

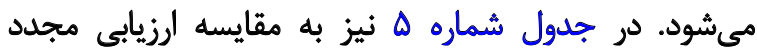

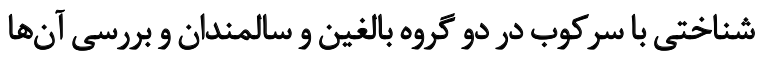

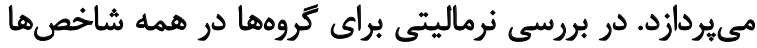

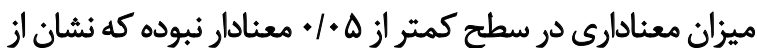
عدم انحراف از فرض نرماليتى را ميدهد أهد. بر اساس يافتههاى ارائه شده در جدول شماره f به منظور برورسى

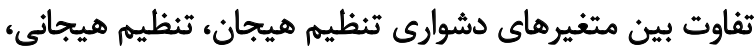

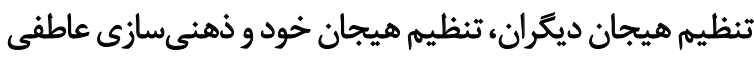

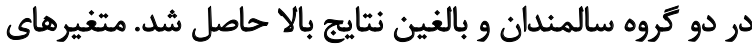

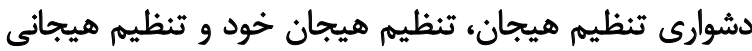

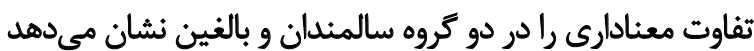

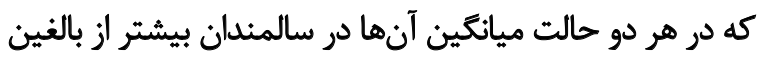

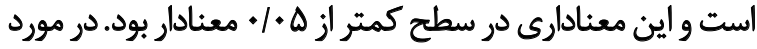

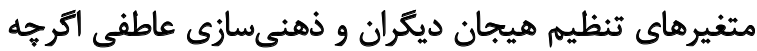

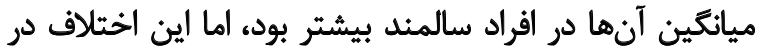

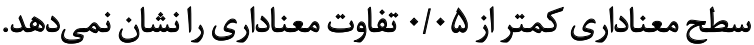

با استناد به جدول شماره ها و بر اساس نتايج حاصل ميزان

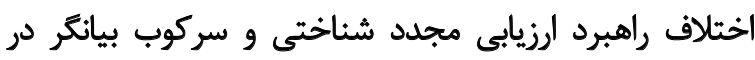

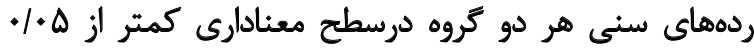

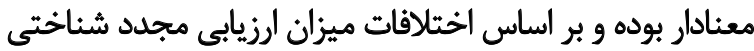
در هر دو رده سنى بيشتر از ميزان سركوب به دسان دست آمده است.

اين مطالعه با هدف مقايسه وضعيت تنظيم هيجان در
دارد. بررسى ساختار عاملى نشان داد كـــهـ هر هـهار مقيـاس

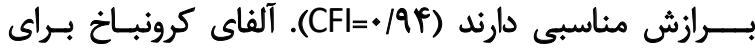

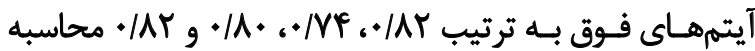

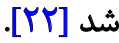

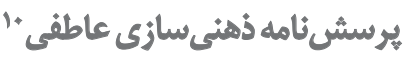

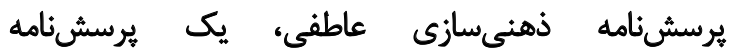

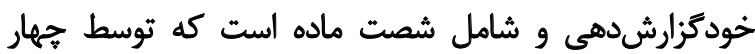

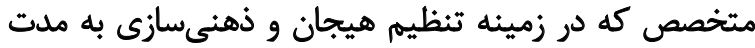

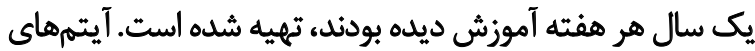

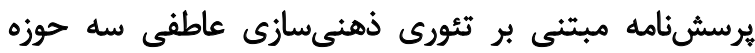

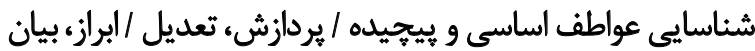

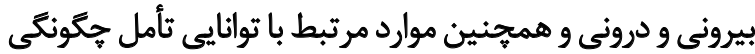

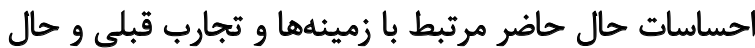

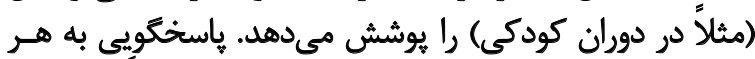

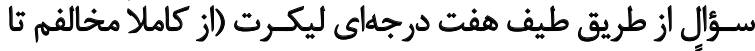

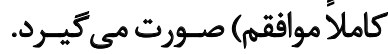

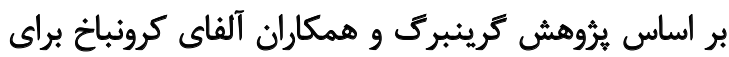

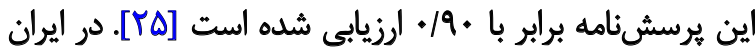

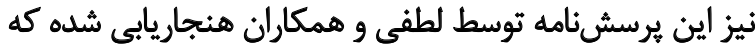

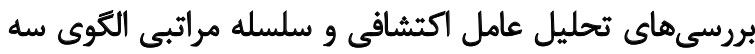

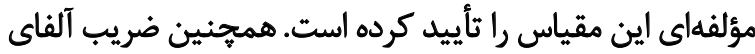

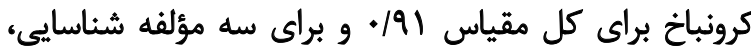

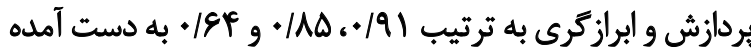

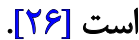

يافتهها

در جدول شماره ا به ارائه اطلاعات جمعيتشناختى به صورت

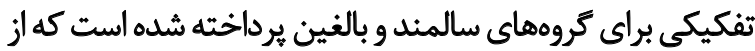

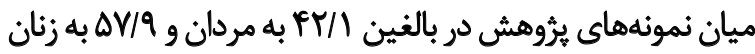

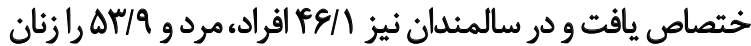

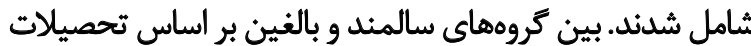

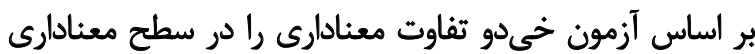

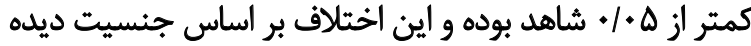

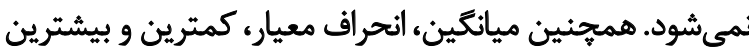

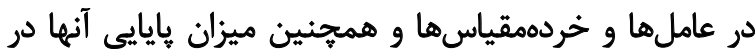
جدول شماره ب برداخته شده است.

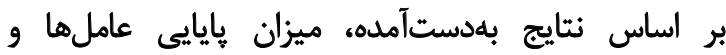

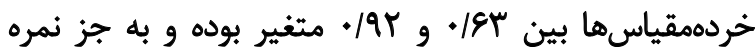

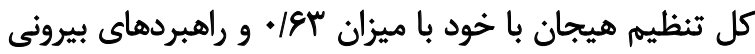

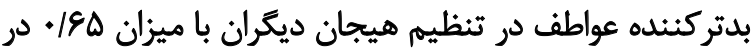


جدول ا. اطلاعات جمعيتشناختى تحصيلات و جنسيت بر اساس تفكيك تروههاي سنى (تعداد نمونهها=هYT)

\begin{tabular}{|c|c|c|c|c|}
\hline \multirow{2}{*}{ أزمون همكنى خى سطو معنادارى } & \multicolumn{2}{|c|}{ فراوانى (درصد) } & \multirow{2}{*}{\multicolumn{2}{|c|}{ مثغير }} \\
\hline & سالمندان & بالفين & & \\
\hline \multirow{5}{*}{$<+1+\infty$} & rAPrE/A) & $\cdot(\cdot)$ & زير دييله & \multirow{5}{*}{ تحصيلات } \\
\hline & $M(M / N)$ & $r \Delta(1 \Delta / V)$ & دييلم & \\
\hline & $1 \cdot(1 M / T)$ & $r \cdot(I r / 9)$ & فوق دييلم & \\
\hline & $M(r / V)$ & $\operatorname{er}(r q)$ & كارشناسى & \\
\hline & $r(Y / Y)$ & $\operatorname{ar}(M T / V)$ & كارشناسي ارشُد و دكترا & \\
\hline \multirow{2}{*}{$\cdot / \Delta V$} & $r \Delta(F \& / 1)$ & $E V(F T / 1)$ & مردان & \multirow[b]{2}{*}{ جنسيت } \\
\hline & $f(\Delta r / q)$ & $\operatorname{qr}(\Delta V / Q)$ & زنان & \\
\hline
\end{tabular}

از اطلاعات مثبت هاسخ دهد. در واقع، كمبود عواطف مثبت و

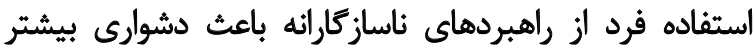

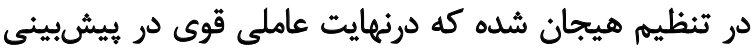

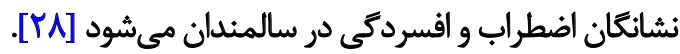

در اين راستا ابروزى و همكاران [YV] نيز در يثوهش خون هود

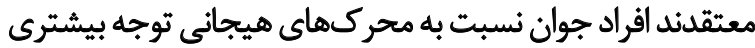

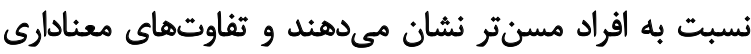

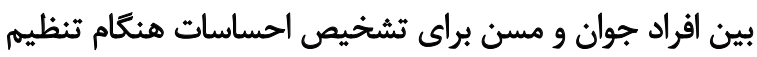
عملكرد شناختي وجود دارد.

باتوجه به اينكه يردازش عاطفى و توجه شامل فعال سازى برخى مئى

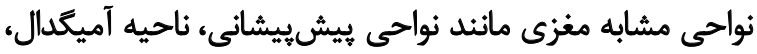

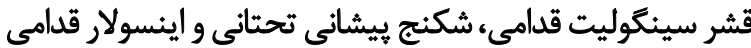

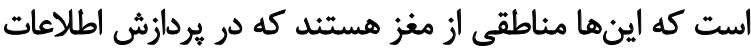

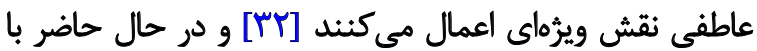

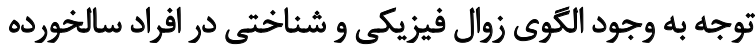

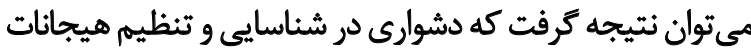

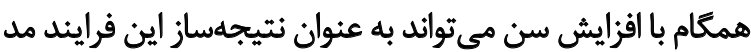
نظر قرار كيرد.

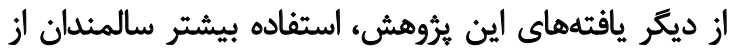

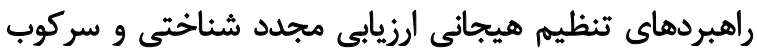

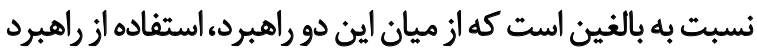

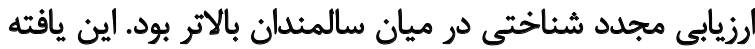

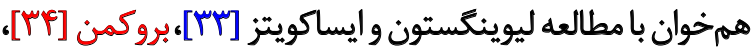

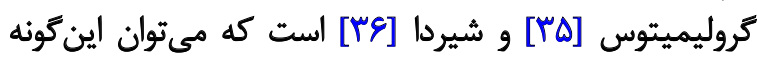

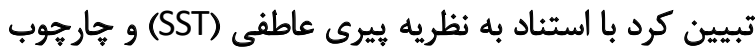

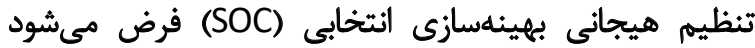

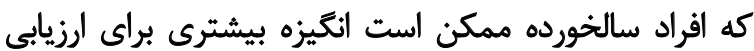

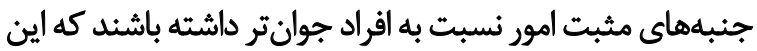

سالمندان و بالغين شهر تهران انجام شد.نتايج بهدست آمده نشان

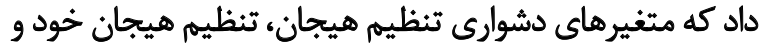

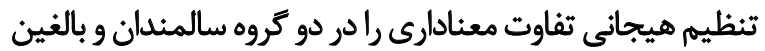

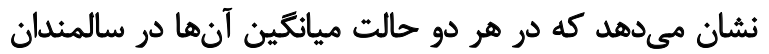

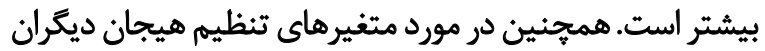

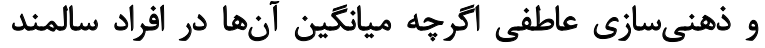

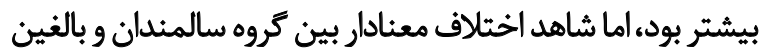

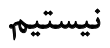
با توجه به يافتههاي بهدستآمده در دشوارى تنظيم هيجان بين

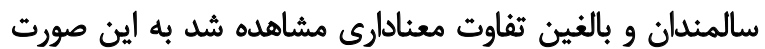

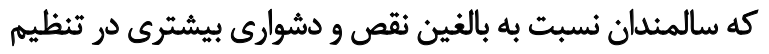

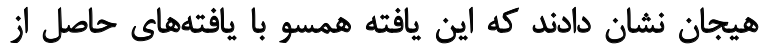

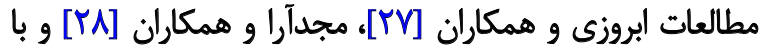

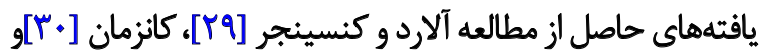

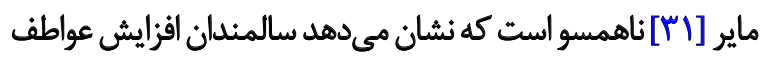

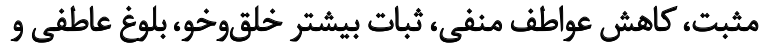

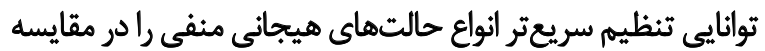

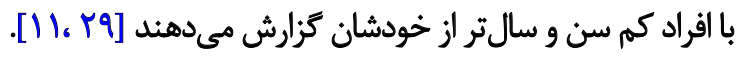
همجنين يُؤهشها نشان دادهاند كه افراد با افزايش سن انغيزه

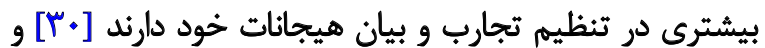

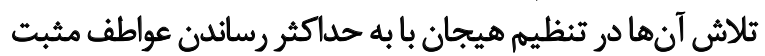

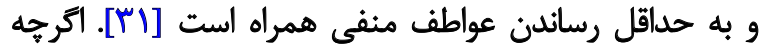

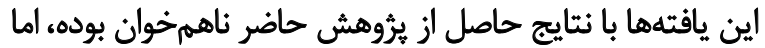

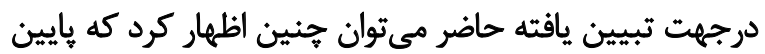

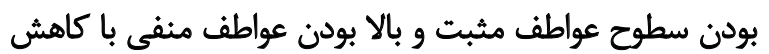

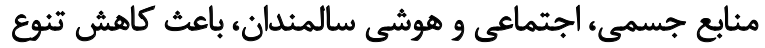

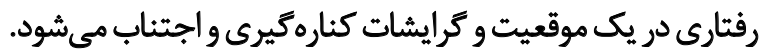
علاوه بر اين سبب مىشود كه فرد به اطلاعات منفى بيش 
جدول ז. اطلاعات توصيفى و شاخص يايايى آلفاي كرونباخ عاملها و خردهمقياسها به تفكيك بالغين و سالمندان

\begin{tabular}{|c|c|c|c|}
\hline \multirow{2}{*}{ ألفاى كروئباخ } & \multicolumn{2}{|c|}{ ميانكين \|نحراقمعيار } & \multirow{2}{*}{ عاملها وخردمدمقياسها } \\
\hline & سالمندان (V) & بالغين (1ه9) & \\
\hline .194 & $9 \Delta / 1 \cdot \pm 1 V / 19$ & AF/erțr+l+r & دشوارى ثنظيم هيجان (ثمره كل) \\
\hline.$/ A F^{P}$ & $\mid \Delta / \cdot \Delta \pm T / M^{e}$ & $|r / T \cdot \pm r / A|$ & عدم يذيرش هاستخ هاي هيجائى \\
\hline$\cdot / A F$ & IQ/ $\mathbf{H}^{\omega} \pm \mathrm{r} / A Y$ & $\mid r \pm F / 18$ & دشوارى در انجام رقفتار هدفمند \\
\hline$\cdot(A)$ & $\mid V / \varepsilon \Delta \pm Y / F e$ & $1 f / e t \pm f /$ R & دشوارى در كتّل تكائه \\
\hline$\cdot M$ & $1 F / M \pm \pm r / 19 q$ & $|\otimes / \&| \pm T / q \Delta$ & فقلان أكاهي هيجانى \\
\hline$\cdot 11$ & $r+/ P Y \pm / q$. & $|V / 8 \Lambda \pm ه / q|$ & دسترسى محلدود به راهبردهاى تنظيم هيجانى \\
\hline -NY & $|r / r| \pm T / 4$. & $1 . / F q \pm r / \Delta F$ & علم وضوح هيجانى \\
\hline$\cdot / 1 \Delta$ & $F / V E \pm . / 9 V$ & $r / 19 \pm 1 / r$ & تنظيم هيجانى (نمره كل) \\
\hline - MA & $f / 99 \pm \cdot / A f$ & $\varphi / \Delta r \pm I / T r$ & 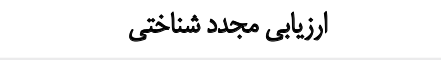 \\
\hline . I I & $f / f I \pm 1 / \Delta)$ & $r / 8 \lambda \pm 1 / r \mu$ & سركوب بيانكر \\
\hline$\cdot / 1$ & $r / \cdot v \pm \cdot / f q$ & $\Psi / * \pm+/ q$ & تنظيم هيجان ديكران (نمره كل) \\
\hline .19 & $H \cdot f \pm \cdot N G$ & $r / A 9 \pm \cdot / N$ & راهبردهاي بيرونى بهبود عواطف \\
\hline .180 & $M / N \pm \star / M T$ & $1 / T+ \pm+/ \Delta F$ & راهبردهاى بيرونى بدتركنتده عواطف \\
\hline.$/ 94$ & $r / W \pm \cdot / T+$ & $r / g r \pm+/ \Delta r$ & تنظيم هيجان خود (نمره كل) \\
\hline -NO & r/gat./81 & $r / \Delta r \pm \cdot / 8$ & راهبردهاي دروئي جهبود عو|طف \\
\hline - RAT & $1 / 49 \pm+184$ & $1 / \pi T \pm+/ 9 F$ & راهبردهاي دروتى بدتر كننده عواطف \\
\hline+191 & $r / q \mu \pm+/ R T$ & F/AF $\pm=/ 8$. & ذهنى سازى عاطلقي (نمره كل) \\
\hline$\cdot / 9$ & $\Delta / \& T \pm+/ \Delta$ & $\Delta / T+ \pm+/ M$ & ن امكذارى هيجانات \\
\hline .191 & $r / q u \pm+/ W$ & $r / A V \pm+/ A \Delta$ & يردازش هيجان \\
\hline$\cdot M^{e}$ & $M / \Delta A \pm \cdot M T$ & $\mathrm{r} / \mathrm{AV} \pm$./Vq & ابراز هيجان \\
\hline
\end{tabular}

L

خود بود كه شاهد تفاوت معنادارى در سالمندان نسبت به بالغين بوديم.

به اين معنا كه در مطالعه حاضر راهبردهاي درونى بهبود عواطف دونف

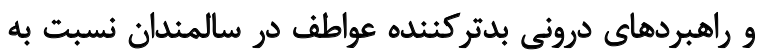

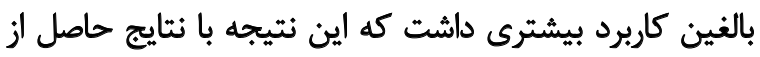

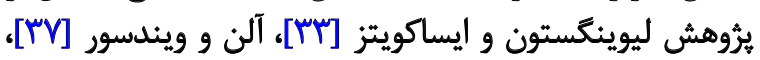

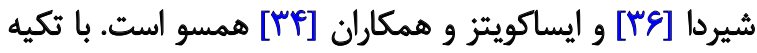

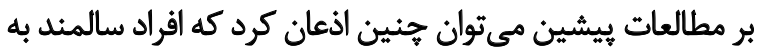

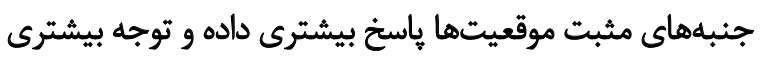

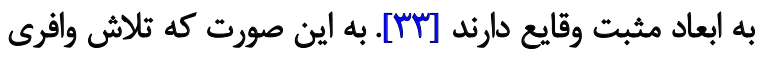

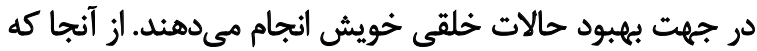

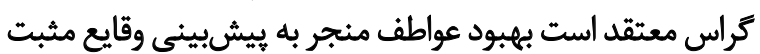

حالت بيانكر نقطه قوتى در سنين سالمندى محسوب مي شيود كه

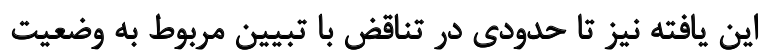

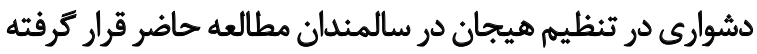

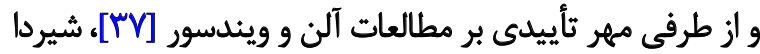

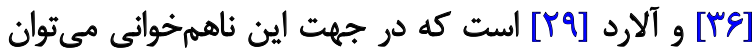

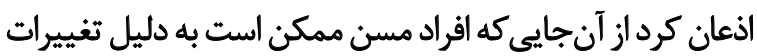

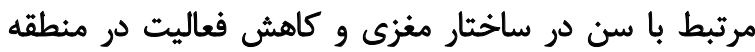

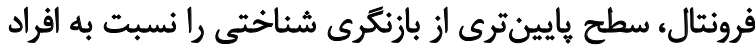

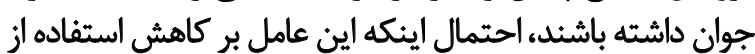

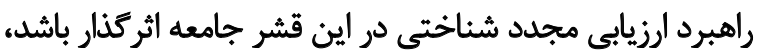
وجود دارد [IT] يافته ديكر مطالعه حاضر ، استفاده از راهبردهاي تنظيم هيجان 
جدول ץ :ضريب همبستكى ييرسون بين عاملها

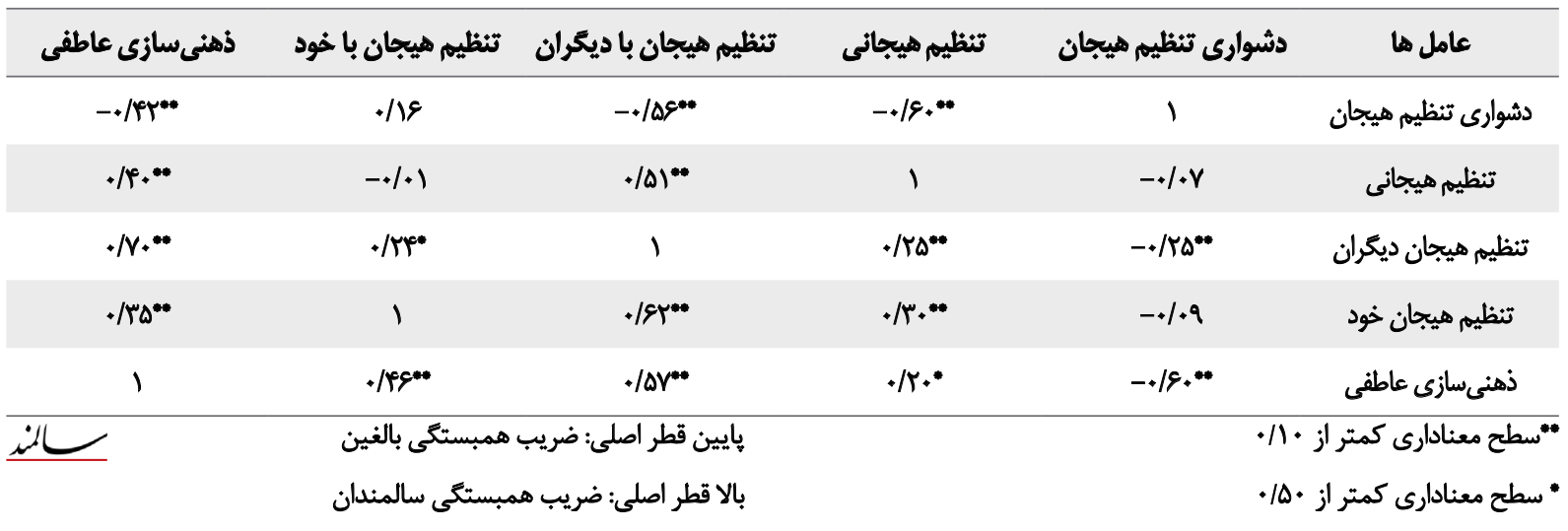

ديدكاه محدوديت زمانى به منظور مديريت بهتر روابط بين فردى

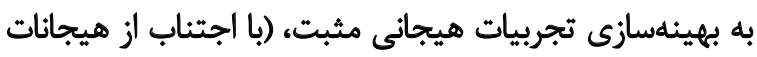

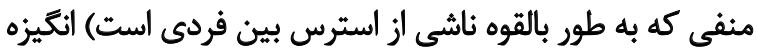
بيشترى دارند [N/] نيز سازكار است.

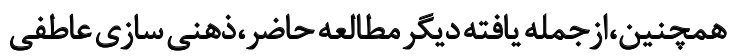

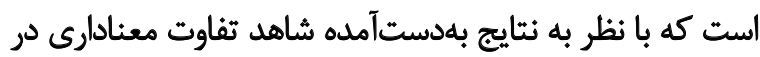

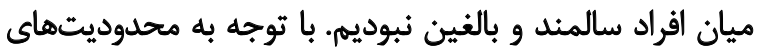

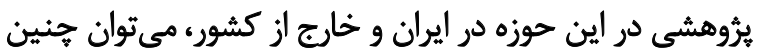

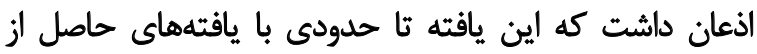

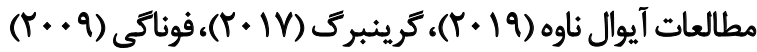

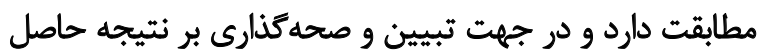

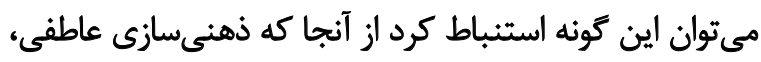

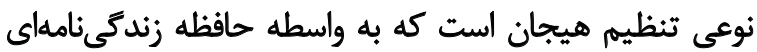

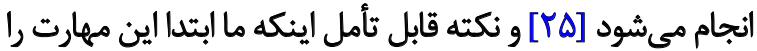

آينده، لذت بردن از رويدادها يا موقعيتهاي مثبت فعلى و يادآورى

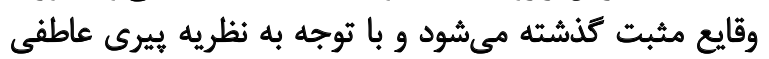
و جارجوب تنظيم هيجانى بهينهسازى انتخابي (SOC)

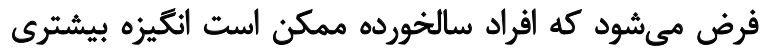

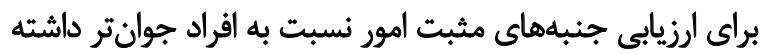

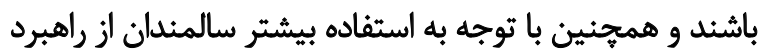

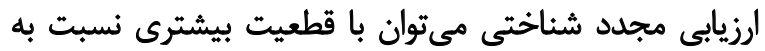

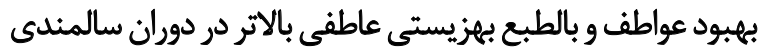

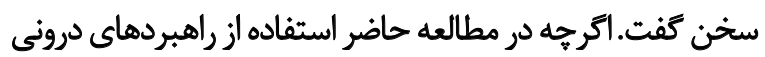

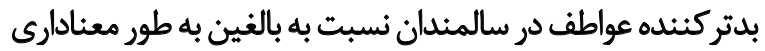

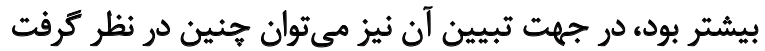

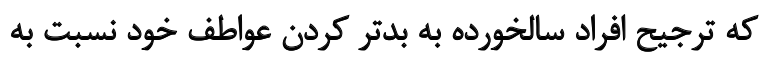

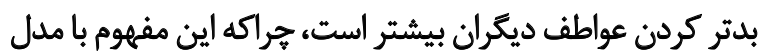

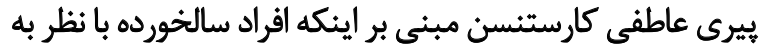

جدول P. نتايج تحليل مقايسهاى براى دشوارى تنظيم هيجان، تنظيم هيجانى، تنظيم هيجان ديكران، تنظيم هيجان خود و ذهنى سازي عاطفى

\begin{tabular}{|c|c|c|c|c|c|c|}
\hline نوع آزمون & معاداري & حد بالا فاصله اطمينان هو & اطمينان هإين فاصله درصد & ميانكين Iانحراف معيار & رده سنى & شاخص \\
\hline \multirow{3}{*}{ أناليز واريانس جند متغيره } & $<+1 .+1$ & $-\Delta / A F$ & $-18 / 2 q$ & $\begin{array}{l}\Lambda r / \& r \pm r \cdot 1 \cdot r \\
q \Delta / \Lambda+ \pm \mid v / \wedge q\end{array}$ & سالمين & هيجان دنظواريم \\
\hline & $<+1 . .1$ &.$- / M A$ & $-\bullet / A f$ & $\begin{array}{l}P / / q \pm 1 / * r \\
r / V E \pm+/ q Y\end{array}$ & بالمبنين & تنظيم هيجانى \\
\hline & .1814 &.$M$ &.$- / 19$ & $\begin{array}{l}r /+r \pm+/ \varepsilon+ \\
r / * v \pm+/ q q\end{array}$ & بالمين بالمثان & تنظيم هيجان \\
\hline \multirow{2}{*}{ تى تست مستقل } & 1.ra & $-* 1 \cdot 1$ & $-\cdot / \pi$ & $\begin{array}{l}r / g Y \pm \cdot / \Delta V \\
r / W \pm \cdot / T \cdot\end{array}$ & بالغين & تنظيم هيجان خود \\
\hline & .114 & $1+4$ & $-\pi / \pi r$ & $\begin{array}{l}f / A f \pm \cdot / 8 . \\
f / q r \pm \cdot / R r\end{array}$ & سالميندان & ذهنى سازى عاطفى \\
\hline
\end{tabular}


جدول ه. نتايج تحليل تى تست زوجى راهبرد ارزيابي مجدد شناختى و سركوب بيائكر

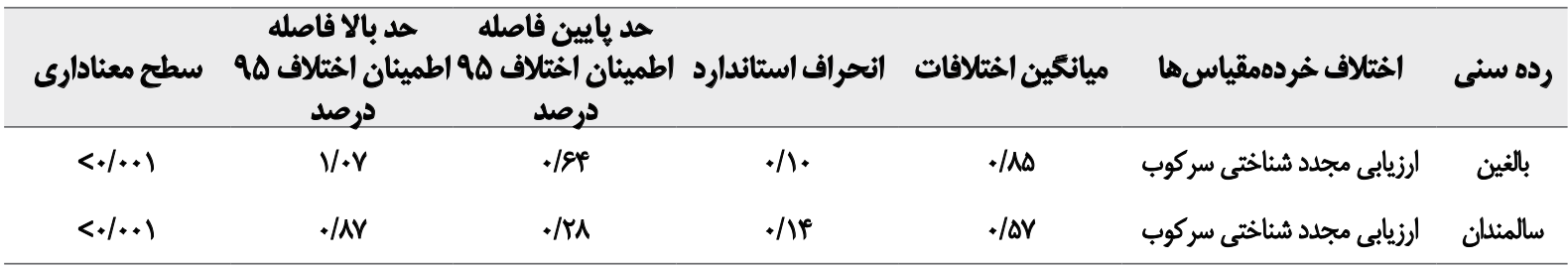

㐾

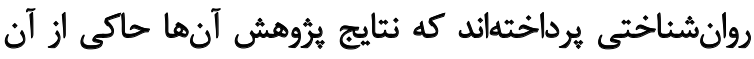

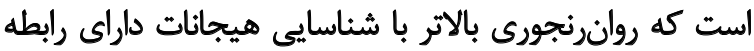

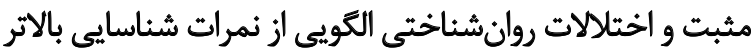

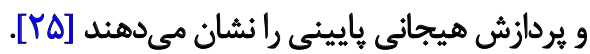

بنابراين بانظر به موارد اشارهشده و همجنين به به اعتقاد فوناكى و

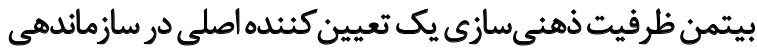

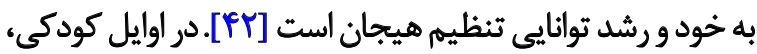

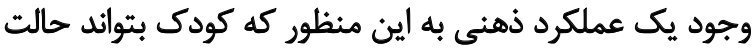

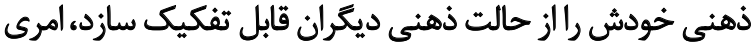

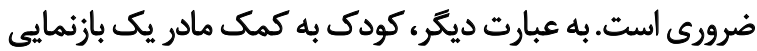

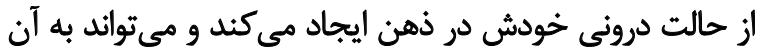

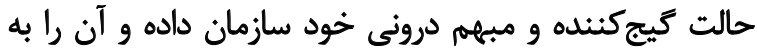

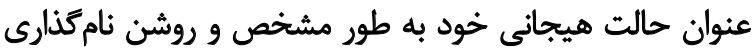

كند [FV]

به عبارتى در تببين ارتباط بين كاركرد كلى خانوادهو مهارتهاي

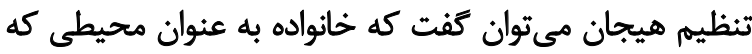

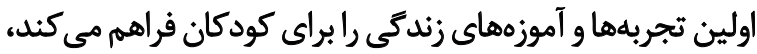

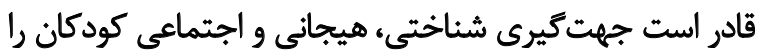

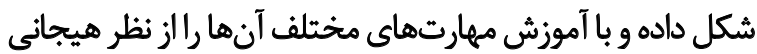

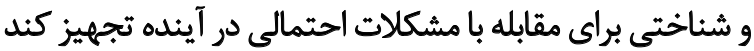

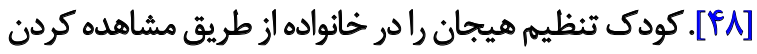

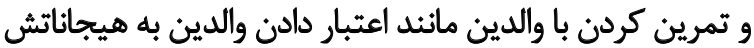

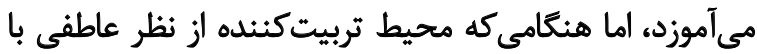

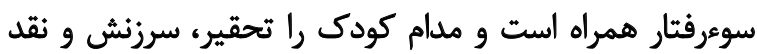

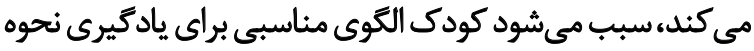

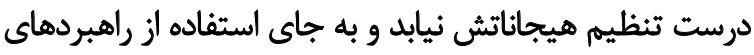

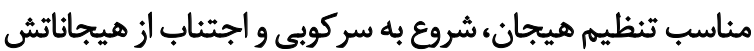

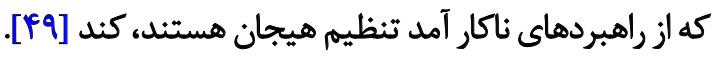

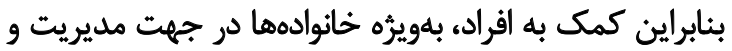

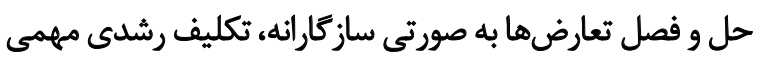

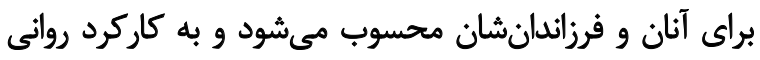
اجتماعى آنها مرتبط است.

\section{نتيجليَيرى نهايى}

در مطالعه حاضر ميزان دشوارى تنظيم هيجان، تنظيم هيجان

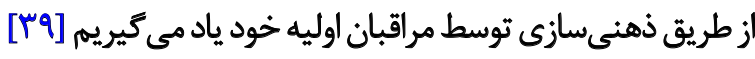

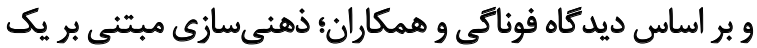

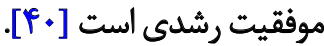

همجنين با نظر به اين امر كه در دوران كودكى؛ شكل ذئيرى

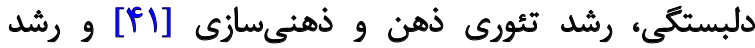

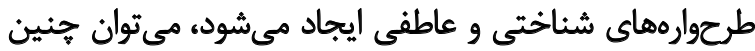

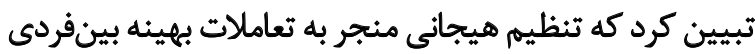

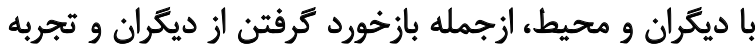

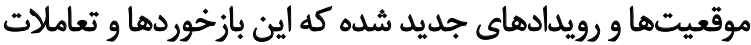

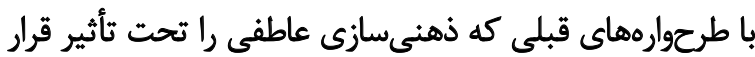

دادهاند، ادغام شده است [بهارئ.

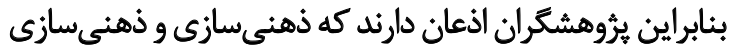

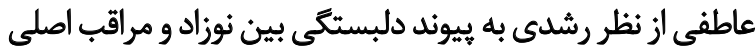

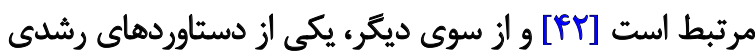

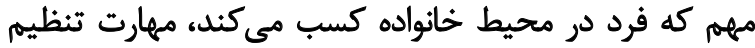

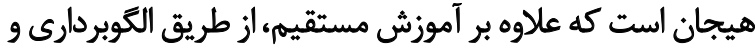

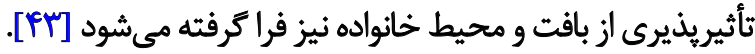

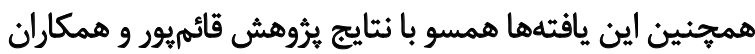

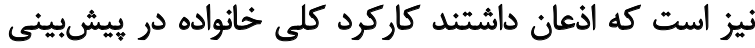

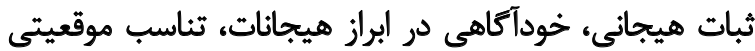

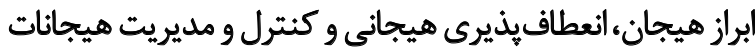

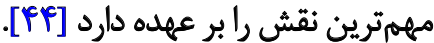

از طرف ديكر، با توجه به اينكه در يُروهش حاضر، شاهد

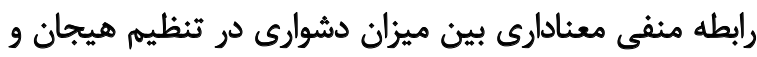

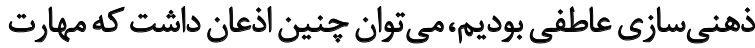

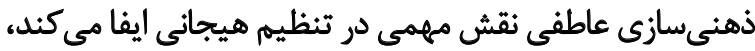

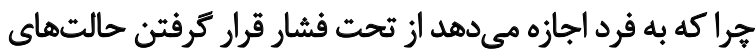

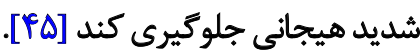

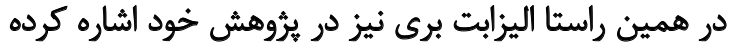

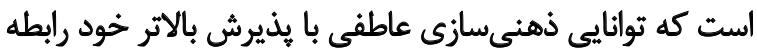

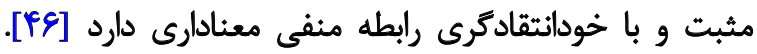

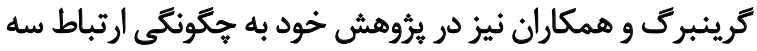

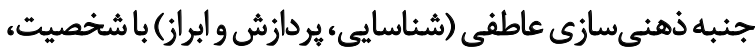

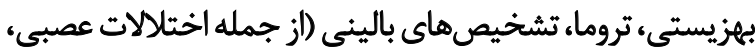

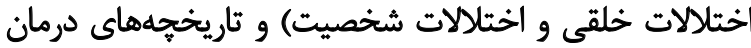




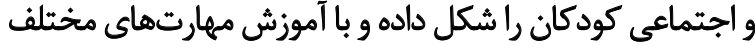

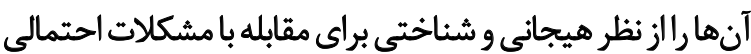

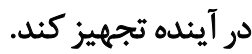

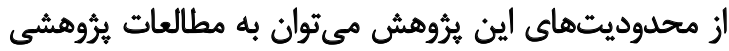

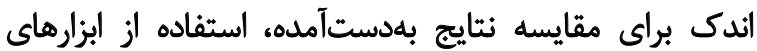

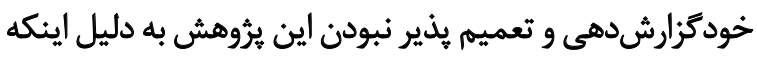

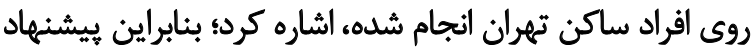

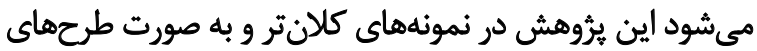

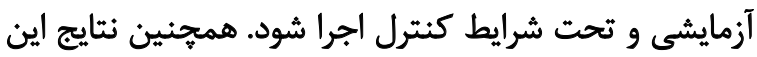

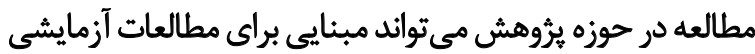

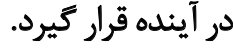

ملاحظات اخلاقي

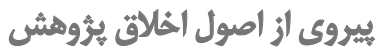

يُروهش حاضر مورد تأييد كميته اخلاق دانشعاه علوم يزشكى

ايران قرار كرفته است (كد: IR.IUMS.REC.1398.955).

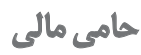

اين تحقيق هيج كونه كمك مالى از سازمان هاى تأمين مالى در

بخش هاى عمومى، تجارى يا غيرانتفاعى دريافت نكرد.

مشاركت نويسند مكان

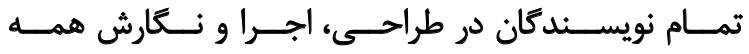

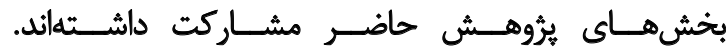

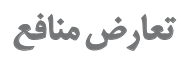

بنابر اظهار نويسندكان اين مقاله تعارض منافع ندارد.
خود و راهبرد ارزيابى مجدد شناختى در سالمندان بالاتر كَزارش

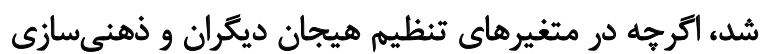

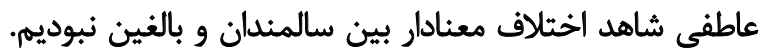

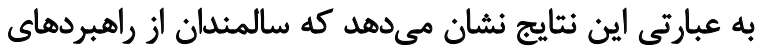

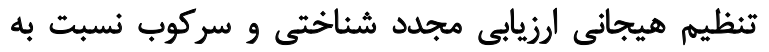

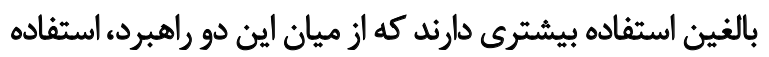
از راهبرد ارزيابى مجدد شناختى در ميان سالمندان بالاتر بود.

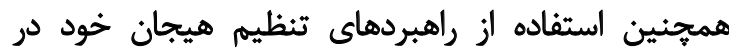

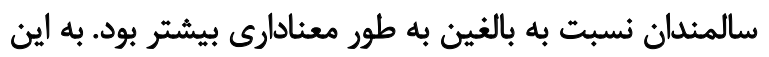

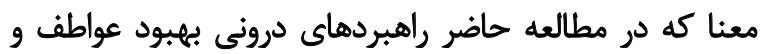

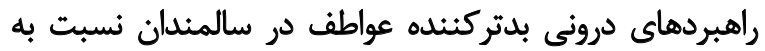

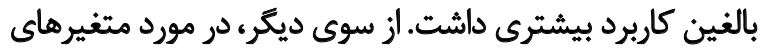

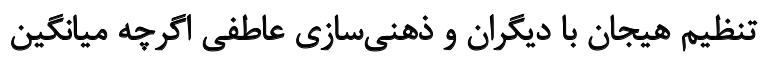

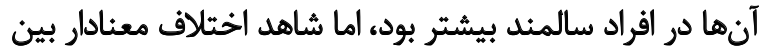
كروه سالمئدان و بالغين نبوديم.

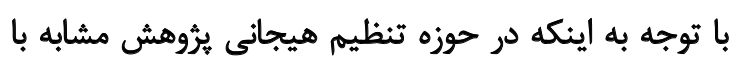

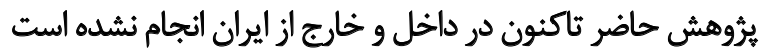

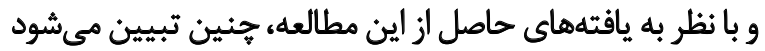

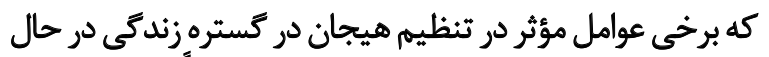

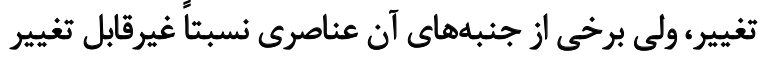
هينتيند

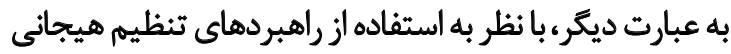

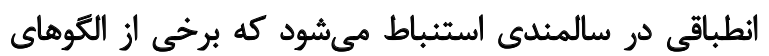

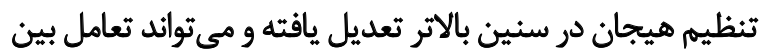

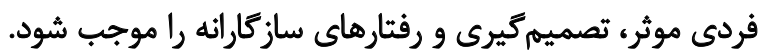

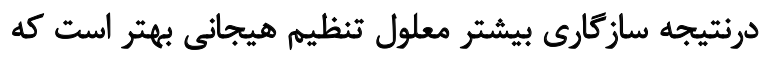

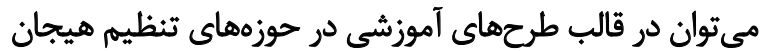

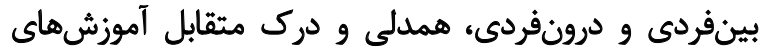

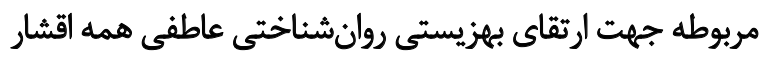
جامعه، على الخصوص افراد جوانتر نيزيز لحاظ شودي.

از سوى ديكر، با توجه به يافتههاى حاصل، به نظر مير برسد افراد

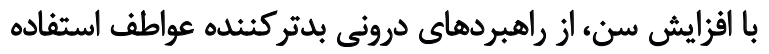

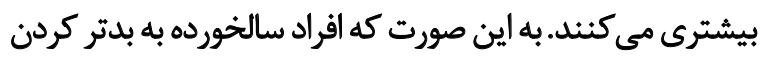

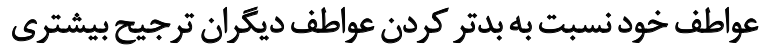

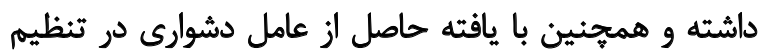
هيجان بيشتر در سالمندان مطالعه حاضر مطابقت المانه دارد.

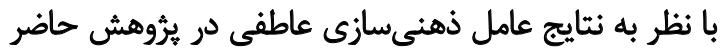

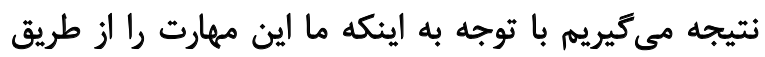

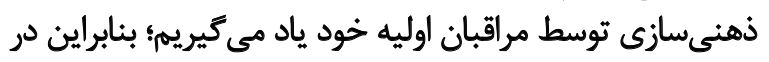

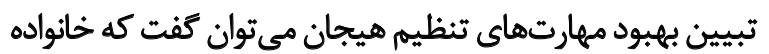

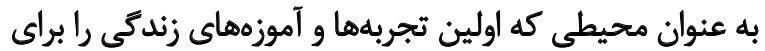
كودكان فراهم مى كند، قادر است جهت تهيرى شناختى، هيجانى 


\section{Refrences}

[1] Hosseinizare SM, Tajvar M, Abdi K, Esfahani P, Geravand B, Pourreza A. [Leisure spending patterns and their relationship with mental health in the elderly in Iran (Persian)]. Salmand: Iranian Journal of Ageing. 2020; 15(3):366-79. [DOI:10.32598/ sija.15.3.2704.1]

[2] Joghataei MT, Nejati V. [Assessment of health status of elderly people in the city of Kashan (Persian)]. Salmand: Iranian Journal of Ageing. 2006; 1(1):3-10. http://salmandj.uswr.ac.ir/article1-26-en.html

[3] Salavati B. [Iran migration outlook 2020 (Persian)]. Tehran: VicePresidency for Science and Technology. http:/ /opac.nlai.ir/opacprod/bibliographic/6778882

[4] Imanzadeh A, Hamrahzdeh M. [Identification of facilitators and deterrents of the quality of life in elderly women and men: A phenomenological research (Persian)]. Salmand: Iranian Journal of Ageing. 2018; 12(4):430-45. [DOI:10.21859/sija.12.4.430]

[5] Khalvati M, Babakhanian M, Khalvati M, Nafei A, Khalvati M, Ghafuri R. [Death anxiety in Iranian elderly: A systematic review and meta-analysis (Persian)]. Salmand: Iranian Journal of Ageing. 2021; 16(2):152-71. [DOI:10.32598/sija.16.2.862.2]

[6] Suárez-Salazar JV, Fresán-Orellana A, Saracco-Álvarez RA. Facial emotion recognition and its association with symptom severity, functionality, and cognitive impairment in schizophrenia: Preliminary results. Salud Mental. 2020; 43(3):105-12. [DOI:10.17711/ SM.0185-3325.2020.015]

[7] Pachankis JE, Cochran SD, Mays VM. The mental health of sexual minority adults in and out of the closet: A populationbased study. Journal of Consulting and Clinical Psychology. 2015; 83(5):890-901. [DOI:10.1037/ccp0000047] [PMID] [PMCID]

[8] Goodall K. Individual differences in the regulation of positive emotion: The role of attachment and self esteem. Personality and Individual Differences. 2015; 74:208-13. [DOI:10.1016/j. paid.2014.10.033]

[9] Mennin DS, Fresco DM, Ritter M, Heimberg RG. An open trial of emotion regulation therapy for generalized anxiety disorder and cooccurring depression. Depression and Anxiety. 2015; 32(8):61423. [DOI:10.1002/da.22377] [PMID] [PMCID]

[10] Kashdan TB, Rottenberg J. Psychological flexibility as a fundamental aspect of health. Clinical Psychology Review. 2010; 30(7):865-78. [DOI:10.1016/j.cpr.2010.03.001] [PMID] [PMCID]

[11] Urry HL, Gross JJ. Emotion regulation in older age. Current Directions in Psychological Science. 2010; 19(6):352-7. [DOI:10.1177/0963721410388395]

[12] Nielsen L, Knutson B, Carstensen LL. Affect dynamics, affective forecasting, and aging. Emotion. 2008; 8(3):318-30. [DOI:10.1037/1528-3542.8.3.318] [PMID] [PMCID]

[13] Charles ST, Piazza JR, Luong G, Almeida DM. Now you see it, now you don't: Age differences in affective reactivity to social tensions. Psychology and Aging. 2009; 24(3):645-53. [DOI:10.1037/ a0016673] [PMID] [PMCID]

[14] Cacioppo JT, Hawkley LC, Kalil A, Hughes ME, Waite L, Thisted RA. Happiness and the invisible threads of social connection: The Chicago health, aging, and social relations study. In: Eid M, Larsen RJ, editors. The Science of Subjective Well-Being. New York: Guilford Publications; 2008. pp. 195-219. https://www.
google.com/books/edition/The_Science_of_Subjective_Well_ Being/uoD1Ly9CeRAC?hl=en\&gbpv $=0$

[15] Stawski RS, Sliwinski MJ, Almeida DM, Smyth JM. Reported exposure and emotional reactivity to daily stressors: The roles of adult age and global perceived stress. Psychology and Aging 2008; 23(1):52-61. [DOI:10.1037/0882-7974.23.1.52] [PMID] [PMCID]

[16] Gross JJ. Emotion regulation: Current status and future prospects. Psychological Inquiry. 2015; 26(1):1-26. [DOI:10.1080/1047 840X.2014.940781

[17] Jurist EL. Mentalized affectivity. Psychoanalytic Psychology. 2005; 23(2):426-44. [DOI:10.1037/0736-9735.22.3.426]

[18] Verzeletti Ch, Zammuner VL, Galli C, Agnoli S. Emotion regulation strategies and psychosocial well-being in adolescence. Cogent Psychology. 2016; 3(1):1199294. [DOI:10.1080/23311908.201 6.1199294]

[19] Charan J, Biswas T. How to calculate sample size for different study designs in medical research? Indian Journal of Psychological Medicine. 2013; 35(2):121-6. [DOI:10.4103/0253-7176.116232] [PMID] [PMCID]

[20] Cohen J. Statistical power analysis for the behavioral sciences Mahwah, NJ: Lawrence Erlbaum Associates; 1988. http:/ /www. utstat.toronto.edu/ brunner/oldclass/378f16/readings/CohenPower.pdf

[21] Choubdar M, Babapour Kheiradin J, Khanjani Z, Zamini S [Relationship between brain-behavioral systems and some demographic factors with cardio-vascular disease (Persian)]. Journal of Applied Psychology. 2011; 5(3):24-36. https://apsy.sbu.ac.ir/ article_95441.html

[22] Lotfi M, Shiasy Y, Amini M, Mansori K, Hamzezade S, Salehi $\mathrm{A}$, et al. [Investigating psychometric properties of Interpersonal Emotion Regulation Questionnaire (IERQ) in university students (Persian)]. Journal of Mazandaran University of Medical Sciences. 2020; 30(185):74-85. http://jmums.mazums.ac.ir/article1-14163-en.htm

[23] Narimani M, Ariapouran S, Abolghasemi A, Ahadi B. [Effectiveness of mindfulness and emotion regulation on physical and psychological well-being among chemical victims (Persian)]. Journal of Kermanshah University of Medical Sciences. 2012; 15(5):e79021. https://sites.kowsarpub.com/jkums/articles/79021.html

[24] Taherifard M, Mikaeili N. [The effectiveness of cognition-based mindfulness therapy on social anxiety, resilience and emotion regulation in women victims of domestic violence (Persian)] Thoughts and Behavior in Clinical Psychology. 2019; 14(51):17-26 https://jtbcp.riau.ac.ir/article_1480_en.html

[25] Greenberg DM, Kolasi J, Hegsted CP, Berkowitz Y, Jurist EL. Mentalized affectivity: A new model and assessment of emotion regulation. PloS One. 2017; 12(10):e0185264. [DOI:10.1371/journal.pone.0185264] [PMID] [PMCID]

[26] Lotfi M, Fard SAS, Amini M. [Adaptation and Investigation the psychometrics characteristics of the Persian version of the mentalized affectivity scale (Persian)]. 2020

[27] Abbruzzese L, Magnani N, Robertson IH, Mancuso M. Age and gender differences in emotion recognition. Frontiers in Psychology. 2019; 10:2371. [DOI:10.3389/fpsyg.2019.02371] [PMID] [PMCID] 
[28] Majad-Ara E, Bigdeli I, Najafi M, Rashn Sh. [Direct and indirect impact of effects on anxiety and depression in elderly: The mediating role of difficulty in emotion regulation (Persian)]. Journal of Clinical Psychology. 2016; 8(2):89-98. [DOI:10.22075/ JCP.2017.2239]

[29] Allard ES, Kensinger EA. Cognitive emotion regulation in adulthood and old age: Positive gaze preferences across two strategies. Aging, Neuropsychology, and Cognition. 2018; 25(2):21330. [DOI:10.1080/13825585.2017.1279265] [PMID]

[30] Kunzmann U, Kupperbusch CS, Levenson RW. Behavioral inhibition and amplification during emotional arousal: A comparison of two age groups. Psychology and Aging. 2005; 20(1):144-58. [DOI:10.1037/0882-7974.20.1.144] [PMID]

[31] Mayer JD, Caruso D, Salovey P. Emotional intelligence: Theory, findings, and implications. Psychological Inquiry. 2004; 15(3):197215. [DOI:10.1207/s15327965pli1503_02]

[32] Pessoa L, McKenna M, Gutierrez E, Ungerleider LG. Neural processing of emotional faces requires attention. Proceedings of the National Academy of Sciences of the United States of America. 2002; 99(17):11458-63. [DOI:10.1073/pnas.172403899] [PMID] [PMCID]

[33] Livingstone KM, Isaacowitz DM. Age similarities and differences in spontaneous use of emotion regulation tactics across five laboratory tasks. Journal of Experimental Psychology: General. 2019; 148(11):1972-92. [DOI:10.1037/xge0000556] [PMID] [PMCID]

[34] Isaacowitz DM, Toner K, Goren D, Wilson HR. Looking while unhappy: Mood-congruent gaze in young adults, positive gaze in older adults. Psychological Science. 2008; 19(9):848-53. [DOI:10.1111/j.1467-9280.2008.02167.x] [PMID] [PMCID]

[35] Gerolimatos LA, Edelstein BA. Predictors of health anxiety among older and young adults. International Psychogeriatrics. 2012; 24(12):1998-2008. [DOI:10.1017/S1041610212001329] [PMID]

[36] Schirda B, Valentine TR, Aldao A, Prakash RS. Age-related differences in emotion regulation strategies: Examining the role of contextual factors. Developmental Psychology. 2016; 52(9):137080. [DOI:10.1037/dev0000194] [PMID]

[37] Allen VC, Windsor TD. Age differences in the use of emotion regulation strategies derived from the process model of emotion regulation: A systematic review. Aging \& Mental Health. 2019; 23(1):1-14. [DOI:10.1080/13607863.2017.1396575] [PMID]

[38] Carstensen LL. Motivation for social contact across the life span: A theory of socioemotional selectivity. In: Jacobs JE, editor. Nebraska Symposium on Motivation, 1992: Developmental Perspectives on Motivation. University of Nebraska Press; 1993. pp. 209-254. https:// psycnet.apa.org/record/1993-98639-005

[39] Aival-Naveh E, Rothschild_Yakar L, Kurman J. Keeping culture in mind: A systematic review and initial conceptualization of mentalizing from a cross_cultural perspective. Clinical Psychology: Science and Practice. 2019; 26(4):e12300. [DOI:10.1111/ cpsp.12300]

[40] Fonagy P, Luyten P. A developmental, mentalization-based approach to the understanding and treatment of borderline personality disorder. Development and Psychopathology. 2009; 21(4):1355-81. [DOI:10.1017/S0954579409990198] [PMID]

[41] Gergely G, Unoka Z. Attachment and mentalization in humans: The development of the affective self. In: Jurist EL, Slade
A, Bergner Sh, editors. Mind to Mind: Infant Research, Neuroscience and Psychoanalysis. New York: Other Press; 2008. pp. 5087.https://www.google.com/books/edition/Mind_to_Mind/ nQztwAEACAAJ?hl=en

[42] Fonagy P, Gergely G, Jurist EL, Target M. Affect regulation, mentalization and the development of the self. London: Routledge; 2019. [DOI:10.4324/9780429471643]

[43] Thorberg FA, Young RM, Sullivan KA, Lyvers M, Connor JP, Feeney GF. Alexithymia, craving and attachment in a heavy drinking population. Addictive Behaviors. 2011; 36(4):427-30. [DOI:10.1016/j.addbeh.2010.12.016] [PMID]

[44] Ghaempour Z, Esmailian M, Sarafraz MR. [Emotion regulation in adolescents: The predicting role of family function and attachment (Persian)]. Thoughts and Behavior in Clinical Psychology. 2019; 14(51):1-16. https://jtbcp.riau.ac.ir/article_1479. $\mathrm{html}$ ?lang=en

[45] Fossati A, Feeney J, Maffei C, Borroni S. Thinking about feelings: Affective state mentalization, attachment styles, and borderline personality disorder features among Italian nonclinical adolescents. Psychoanalytic Psychology. 2014; 31(1):41-67. [DOI:10.1037/a0033960]

[46] Berry E. Mentalized affect, self-acceptance, and self-criticism [BAs. thesis]. Winter Park, FL: Rollins College; 2019. https:// scholarship.rollins.edu/honors/80/

[47] Slade A, Holmes J. Attachment and psychotherapy. Current Opinion in Psychology. 2019; 25:152-6. [DOI:10.1016/j.copsyc.2018.06.008] [PMID]

[48] Carrère S, Bowie BH. Like parent, like child: Parent and child emotion dysregulation. Archives of Psychiatric Nursing. 2012; 26(3):E23-30. [DOI:10.1016/j.apnu.2011.12.008] [PMID]

[49] Mills P, Newman EF, Cossar J, Murray G. Emotional maltreatment and disordered eating in adolescents: Testing the mediating role of emotion regulation. Child Abuse \& Neglect. 2015; 39:15666. [DOI:10.1016/j.chiabu.2014.05.011] [PMID] 